\title{
Serum Levels of the Inflammatory Cytokines in Patients with Lumbar Radicular Pain Due to Disc Herniation
}

\author{
Bo Zu ${ }^{1,2}$, Hong Pan ${ }^{2}$, Xiao-Jun Zhang ${ }^{2}$, Zong-Sheng Yin ${ }^{3}$ \\ ${ }^{I}$ Department of Orthopaedics, Anhui Medical University, Hefei, China \\ ${ }^{2}$ Department of Orthopaedics, Anqing Hospital, Anhui Medical University, Anqing, China \\ ${ }^{3}$ Department of Orthopaedics, The First Affiliated Hospital of Anhui Medical University, Hefei, China
}

\begin{abstract}
Study Design: Cohort study.
Purpose: This study primarily aimed to evaluate the serum levels of tumor necrosis factor-alpha (TNF- $\alpha$ ) and interleukin (IL)-4 in patients with lumbar radiculopathy 1 and 12 months after microdiscectomy.

Overview of Literature: Lumbar radiculopathy is possibly caused by inflammatory changes in the nerve root. The intraneural application of pro-inflammatory cytokines induces behavioral signs associated with pain. Anti-inflammatory cytokine treatment effectively reduces hyperalgesia.

Methods: The role of TNF- $\alpha$ and IL-4 in long-lasting lumbar radiculopathy was addressed. A total of 262 patients were recruited from Anqing Hospital, Anhui Medical University. During inclusion at 1 and 12 months, serum concentrations of TNF- $\alpha$ and IL-4 were analyzed by enzyme-linked immunosorbent assay, and pain intensity was reported on a 0-10 cm visual analog scale (VAS).

Results: Sixty six patients had VAS $<3$ and 196 patients had VAS $\geq 3$. Serum concentrations of pro-inflammatory TNF- $\alpha$ and antiinflammatory IL-4 in patients with lumbar radiculopathy related to disc herniation were measured at 1- and 12-month follow-up. TNF- $\alpha$ decreased in both VAS groups with time. In contrast, IL-4 increased in both groups at 1 month and then decreased gradually until month 12. The changes in serum levels of TNF- $\alpha$ and IL-4 over time between the VAS $\geq 3$ and VAS $<3$ groups were significantly different.

Conclusions: Chronic lumbar radiculopathy may be associated with high level of pro-inflammatory substances, such as TNF- $\alpha$, in serum after disc herniation, and elevated anti-inflammatory cytokine in patients with lumbar radiculopathy may indicate a favorable outcome.
\end{abstract}

Keywords: Interleukins; Tumor necrosis factor- $\alpha$; Pain; Cytokines

\section{Introduction}

Lumbar radiculopathy is a term that describes symptoms of pain, numbness, and/or weakness that radiate along the sciatic nerve from the lower back to the buttocks and leg [1]. Lumbar radiculopathy is a relevant health problem that affects quality of life, resulting in high health costs and economic loss worldwide [2]. The reported prevalence

\footnotetext{
Received Nov 23, 2015; Revised Feb 21, 2016; Accepted Mar 7, 2016

Corresponding author: Zong-Sheng Yin

Department of Orthopaedics, The First Affiliated Hospital of Anhui Medical University,

218 Jixi Road, Hefei, Anhui 230022, China

Tel: +86-139-5697-7198, Fax:+86-551-343-2123, E-mail: yinzongsheng@sina.com
} 
of sciatica varies widely from $1.2 \%$ and $43 \%$ in the general population [3].

Although initially believed to be a primary mechanical insult to the nerve root and dorsal root ganglion, lumbar radiculopathy is possibly caused by inflammatory changes in the nerve root [4]. The role of cytokinemediated neuroimmune interactions in the development and persistence of pain has been extensively studied [57]. Intraneural application of pro-inflammatory cytokines induces behavioral signs associated with pain [8]. Antiinflammatory cytokine treatment effectively reduces hyperalgesia [9]. Inflammatory cytokine inhibitors provided long-lasting analgesia in an inflammatory neuropathic pain model $[10,11]$. On the basis of these findings, we evaluated whether cytokine profiles differ between severe and mild human sciatica, as well as whether distinct cytokine profiles provide relevant information regarding lumbar radiculopathy pathogenesis.

Tumor necrosis factor-alpha (TNF- $\alpha$ ) is a pleiotropic cytokine that can stimulate inflammatory responses of synapses and myelin sheath, promote cellular apoptosis because of its cytotoxic effect, and induce nerve swelling and neuropathic pain [12]. TNF- $\alpha$ influences neural survival, exerting both neuroprotective and neurodegenerative actions [13]. Following peripheral nerve injury, upregulation of TNF- $\alpha$ expression has been documented in several neuropathic pain models [14]. In contrast, anti-inflammatory cytokines, such as interleukin 4 (IL-4), may inhibit proinflammatory cytokine response. IL-4 may directly interact with the endogenous opioid system by inducing and upregulating the transcription of $\mu$ and $\delta$ opioid receptors, and production of anti-inflammatory cytokines may reflect a natural analgesic system regulating the activity and sensitivity of the endogenous opioid system [15].

Serum levels of inflammatory cytokines in patients over a long period of time are unclear. No clear correlation exists between inflammatory cytokines and the severity of pain after microdiscectomy. The current study primarily aimed to evaluate the serum levels of TNF- $\alpha$ and IL- 4 in patients with lumbar radiculopathy at 1 and 12 months after microdiscectomy.

\section{Materials and Methods}

\section{Participants}

A total of 300 patients with lumbar radiculopathy sec- ondary to disc herniation were recruited from our spine center from 2013 to 2014. The inclusion criteria were age between 18 and 60 years, lumbar disc herniation verified on magnetic resonance imaging (MRI) with dermatomal distribution of pain in lower limb, pain lasting for at least 1 month, and positive straight leg raising test. Exclusion criteria were use of oral or systemic corticosteroid therapy in the preceding 3 months, treatment with acetaminophen or a non-steroidal anti-inflammatory drug, lumbar spinal stenosis, previous surgery for herniated disc at the same level or fusion at any level in lumbar spine, generalized musculoskeletal pain, inflammatory rheumatic disease, diabetic polyneuropathy, cardiovascular disease (NYHA III and IV), cancer, psychiatric disease, cauda equina syndrome, alcohol or drug abuse, recent surgery (within 1 month), pregnancy, or poor Norwegian language. Ten patients $(3.3 \%)$ were excluded with another $20(6.7 \%)$ patients excluded because of missing clinical data at baseline. In addition, 8 patients (2.6\%) dropped out during the study. The final clinical dataset comprised 262 patients. All included patients received microdiscectomy and underwent rehabilitation under the instruction of doctors beginning about one week after surgery.

\section{Ethics statement}

Patients were included after they signed a written informed consent. The study was approved by the Clinical Ethics Committee of Anhui Medical University.

\section{Blood sampling and analysis}

Before blood was withdrawn, all patients completed a pain evaluation questionnaire at baseline and the 12-month follow-up. Venous blood was collected between 8:00 AM and 9:00 AM to reduce variability resulting from diurnal variations. Cytokine concentration in the serum was analyzed. Two $10 \mathrm{~mL}$ blood samples were drawn into glass tubes containing $35 \mu \mathrm{mol}$ of dipotassium ethylenediaminetetraacetic acid and 1,500 kallikrein inactivator units of Trasylol. The tubes were kept in an ice bath and then centrifuged at $2,000 \times \mathrm{g}$ for 15 minutes at $4^{\circ} \mathrm{C}$. Plasma was separated from the cells, stored at $-80^{\circ} \mathrm{C}$, and analyzed using a commercially available enzyme-linked immunosorbent assay (ELISA). Based on the observations in the clinic, we devised a visual analog scale (VAS) method to determine the cutoff that best distinguished patients with 
chronic pain as a function of the impact of pain on patient functioning. A VAS score on a 0-10 point scale of 1-2 indicated no or low pain, 3-6 indicated moderate pain, and $7-10$ indicated severe pain. Presently, scores $\geq 3$ were classified as high pain and $<3$ as low pain.

\section{ELISA}

Protein levels of TNF- $\alpha$ and IL- 4 were determined from the sera of patients with sciatica at 1 and 12 months. Commercial ELISA kits were used in accordance with the manufacturer's instructions (Abcam, Cambridge, UK). The assays were performed in duplicate in 96-well plates, and the results are presented as $\mathrm{pg} / \mathrm{mL}$. The collected samples were analyzed on the same day on one ELISA plate for each cytokine.

\section{Data evaluation and statistical analyses}

Data are shown as mean \pm standard error of the mean. The serum levels of TNF- $\alpha$ and IL- 4 in the high-pain group (VAS $\geq 3$ at 12 -month follow-up) versus the low-pain group (VAS $<3$ at 12 -month follow-up) were compared by repeated measures analysis of variance (rmANOVA). Statistical analyses were performed using SPSS version 18. Unequal variance and sample size were corrected for by the software. A $p$-value $<0.05$ represented the level of statistical significance.

\section{Results}

Data from 262 patients were included in the analyses. On the basis of pain intensity on the 0-10 VAS, two groups were devised: the high pain group (VAS $\geq 3 ; \mathrm{n}=196$ ) and low pain group (VAS <3; $n=66$ ). At baseline, 132 patients $(67 \%)$ in the high pain group were smokers, which was significantly higher than the $20(30 \%)$ smokers in the low pain group. The duration of sciatica in the VAS $\geq 3$ group (mean, 48.10) was significantly longer than in the VAS $<3$ group (mean, 23.4). In the VAS $\geq 3$ group there were 30 protruded, 160 extruded, and 6 sequestrated discs. The respective numbers in the VAS $<3$ group were 46,18 , and 2. There were significant differences in the protruded and extruded types, and Oswestry disability index (ODI) total score preoperatively and at the 12-month follow-up. The initial VAS score in the VAS $\geq 3$ group $(7.8 \pm 0.6)$ was not significantly different from the low pain group $(7.7 \pm 0.6)$. No difference was observed between the VAS $\geq 3$ and VAS $<3$ groups in terms of age, sex, and leg pain (Table 1).

Serum concentrations of pro-inflammatory TNF- $\alpha$ and anti-inflammatory IL-4 in patients with lumbar radiopathy secondary to disc herniation were measured at inclusion

Table 1. Baseline characteristics of patients

\begin{tabular}{|c|c|c|c|}
\hline Characteristic & VAS $\geq 3$ group $(n=196)$ & VAS $<3$ group $(n=66)$ & $p$-value \\
\hline Age (yr) & $34.0 \pm 12.3$ & $35.2 \pm 10.4$ & $0.84^{\mathrm{al}}$ \\
\hline Sex (male/female) & $51 / 49(100 / 96)$ & $50 / 50(33 / 33)$ & $0.89^{b)}$ \\
\hline Smoker (yes/no) & $67 / 33(132 / 64)$ & $30 / 70(20 / 46)$ & $<0.01^{\text {b) }}$ \\
\hline Duration of sciatica (wk) & $48.10 \pm 28.96$ & $23.40 \pm 5.97$ & $<0.01^{\mathrm{c})}$ \\
\hline Leg pain, left-side/right-side (\%) & $50 / 50(98 / 98)$ & $50 / 50(33 / 33)$ & $0.98^{b)}$ \\
\hline \multicolumn{4}{|l|}{ The type of herniated disc (\%) } \\
\hline Protruded type & $30(15)$ & $46(70)$ & $<0.01^{\text {b) }}$ \\
\hline Extruded type & $160(82)$ & $18(27)$ & $<0.01^{\text {bl }}$ \\
\hline Sequestrated type & $6(3)$ & $2(3)$ & $0.67^{b)}$ \\
\hline Visual analog scale & $7.8 \pm 0.6$ & $7.7 \pm 0.6$ & $0.67^{\text {a) }}$ \\
\hline \multicolumn{4}{|l|}{ Oswestry disability index } \\
\hline At baseline (\%) & $70 \pm 19$ & $45 \pm 24$ & $<0.05^{\mathrm{c})}$ \\
\hline At 12-month (\%) & $42 \pm 16$ & $28 \pm 10$ & $<0.05^{\mathrm{c})}$ \\
\hline
\end{tabular}

Values are presented as mean \pm standard deviation or number (\%).

The Oswestry disability index ranges from 0 to 100, with lower scores indicating less severe symptoms.

VAS, visual analog scale.

a)Unpaired Student's $t$ test; ${ }^{\text {bl Pearson chi-square; }{ }^{\text {cl}} T w o-s i d e d ~ M a n n-W h i t n e y ~} U$ test. 
Table 2. The serum concentration of the TNF- $\alpha$ and IL-4 measured at baseline, at 1 month and at 12 months

\begin{tabular}{|c|c|c|c|}
\hline Inflammatory cytokines & VAS $\geq 3$ group & VAS $<3$ group & $p$-value ${ }^{\mathrm{a}}$ \\
\hline \multicolumn{4}{|l|}{ Tumor necrosis factor- $\alpha$ (pg/mL) } \\
\hline Baseline & $123.81 \pm 30.67$ & $80.01 \pm 36.88$ & $<0.001$ \\
\hline $1 \mathrm{mo}$ & $79.53 \pm 34.36$ & $71.78 \pm 31.09$ & 0.02 \\
\hline $12 \mathrm{mo}$ & $75.96 \pm 33.26$ & $69.92 \pm 32.79$ & 0.07 \\
\hline \multicolumn{4}{|l|}{ Interleukin-4 (pg/mL) } \\
\hline Baseline & $3.46 \pm 2.33$ & $5.03 \pm 3.23$ & $<0.001$ \\
\hline $1 \mathrm{mo}$ & $5.09 \pm 2.62$ & $5.41 \pm 3.08$ & $<0.001$ \\
\hline $12 \mathrm{mo}$ & $3.87 \pm 2.24$ & $5.14 \pm 2.99$ & $<0.001$ \\
\hline
\end{tabular}

TNF- $\alpha$, tumor necrosis factor-alpha; IL-4, interleukin 4; VAS, visual analog scale.

a) Unpaired Student's test.

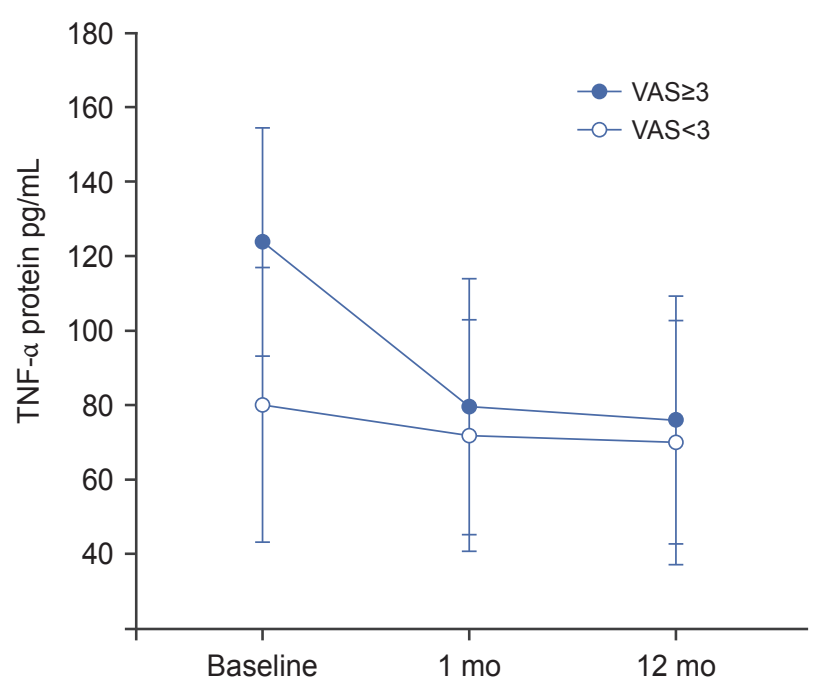

Fig. 1. ODI in the two patient groups. ODI was significantly correlated with TNF- $\alpha$ in the VAS $\geq 3$ group $(r=0.2, p<0.01)$ and VAS $<3$ group $(r=0.37, p<0.01)$ at the 12-month follow-up, but was not significantly correlated with IL-4 in the VAS $\geq 3$ group $(r=-0.09, p=0.23)$ and VAS $<3$ group $(r=0.08, p=0.5)$ at the 12-month follow-up. ODI, Oswestry disability index; TNF- $\alpha$, tumor necrosis factor-alpha; VAS, visual analog scale; IL-4, interleukin 4.

and at 1 - and 12-month follow-up (Table 2). TNF- $a$ decreased over time in the VAS $\geq 3$ and VAS $<3$ groups (Fig. $1)$, while IL-4 increased in both groups at 1 month and then gradually decreased until month 12 (Fig. 2). The changes in serum levels of TNF- $\alpha$ and IL-4 over time between the VAS $\geq 3$ and VAS $<3$ groups were significantly different. ODI was significantly correlated with TNF- $\alpha$ in the VAS $\geq 3$ group $(r=0.2, p<0.01)$ and VAS $<3$ group $(r=0.37, p<0.01)$ at the 12-month follow-up. ODI was not significantly correlated with IL- 4 in the VAS $\geq 3$ group $(r=-0.09, p=0.23)$ and VAS $<3$ group $(r=0.08, p=0.5)$ at the

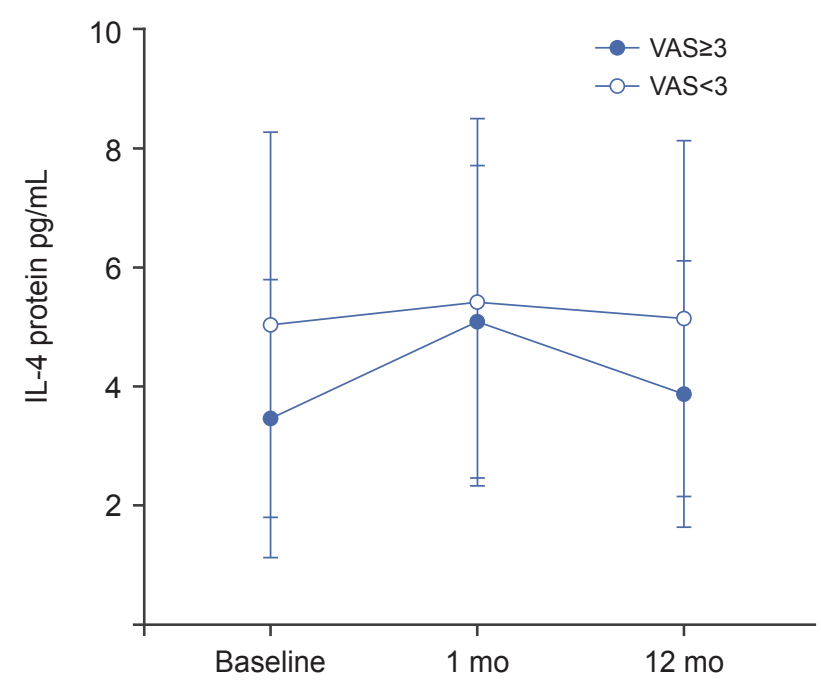

Fig. 2. Serum concentration of the pro-inflammatory cytokines TNF- $\alpha$ in patients with lumbar radicular pain due to disc herniation measured at inclusion, 1 month, and 12 months follow-up. The data are given as mean \pm standard error of the mean. TNF- $\alpha$, tumor necrosis factor-alpha; VAS, visual analog scale; IL-4, interleukin 4.

12-month follow-up (Fig. 3).

\section{Discussion}

This study investigated the involvement of the TNF- $\alpha$ and IL-4 inflammatory cytokines in the serum of patients with lumbar radiopathy. Previous studies have contributed to the understanding of the pro-inflammatory cytokine process in serum during a 1-year follow-up, demonstrating that pro-inflammatory cytokines may be associated with the mechanisms underlying the development of chronic 

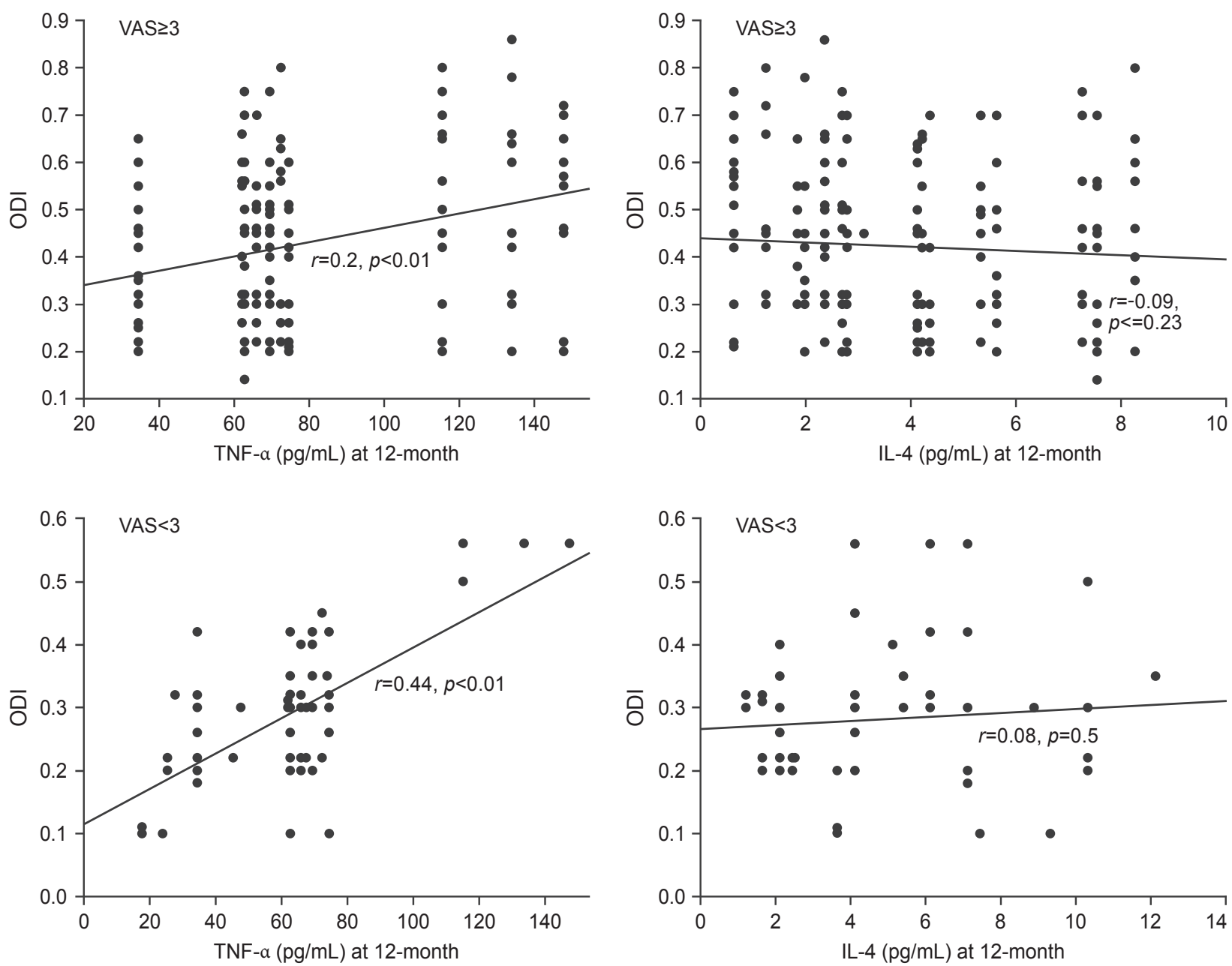

Fig. 3. Serum concentration of the anti-inflammatory cytokine IL-4 in patients with lumbar radicular pain due to disc herniation measured at inclusion, 1 month, and at 12 months follow-up. The data are given as mean \pm standard error of the mean. ODI, Oswestry disability index; TNF- $\alpha$, tumor necrosis factor-alpha; VAS, visual analog scale; IL-4, interleukin 4.

pain after disc herniation, and cytokine reduction during early phase may also be a reflection of treatment [16].

In the present study, TNF- $\alpha$ blood protein levels in the VAS $\geq 3$ group were higher than those in the VAS $<3$ group, while IL- 4 was higher in the VAS $<3$ group. These results are consistent with previous reports and may provide new quantitative evidence regarding high amounts of anti-inflammatory cytokine in patients with lumbar radiculopathy to possibly indicate a favorable outcome. IL-4 upregulates opioid receptors [17] and the increase in IL-4 production may correspond to the natural analgesic system regulating the activity and sensitivity of the endogenous opioid system. IL-4 markedly inhibits the expression and release of pro-inflammatory cytokines. Moreover, IL-4 can block or suppress monocyte-derived cytokines including TNF- $\alpha$, IL-1, IL-6, IL-8, and macrophage inflammatory protein $1 \alpha$. IL-4 can also suppress macrophage cytotoxic activity and macrophage-derived nitric oxide production [18]. In the current work, higher TNF- $\alpha$ blood protein levels were found in the VAS $\geq 3$ group. There are several explanations. First, TNF- $\alpha$ can induce apoptosis in dorsal root ganglion neurons and apoptosis-associated caspase promotes pain-related behavior [19]. Second, TNF- $\alpha$ can give rise to the changes in potassium channel in these neurons and decrease potassium ion outward currents, leading to overall neuronal hyperexcitability and sciatica pain [20].

Significantly more of those in the VAS $\geq 3$ group were TNF- $\alpha$ positive at all three time points during the 12-month follow-up. The TNF- $\alpha$ level in these patients declined during the first month of multidisciplinary treatment but remained high throughout the entire follow-up 
period. TNF- $\alpha$, as a pro-inflammatory mediator, plays a central role in the pathophysiology of lumbar radiculopathy [21]. In the present study, the IL-4 levels in all the patients were also elevated slightly during the first month of multidisciplinary treatment and declined slowly to the 12th month. The subtle process of IL-4 elevation and decline during the follow-up period suggests a protective mechanism that is analgesic for neuropathic pain.

Age, sex, smoking status, duration of lumbar radiculopathy, side of pain, and ODI were comparable in both groups. Inflammatory diseases, advanced degenerative spinal changes, and medications including nonsteroidal anti-rheumatic drugs, and locally or systemically administered corticosteroids that may influence inflammatory processes were excluded in this study. Notably, microdiscectomy in patients with protruded and extruded type was associated with better outcomes than sequestrated type.

There are some limitations. Protein analysis was partially impeded by the low serum levels of some examined cytokines. Negative results were easily obtained because of the limited number of samples. Additional data should be obtained with time to address this issue. Several factors including the type of herniated disc and duration of symptoms affect serum cytokine levels, which could inevitably produce biased results. The concentration of inflammatory cytokines close to the nerve roots or in the cerebrospinal fluid was not measured. Hence, the current results cannot provide direct information regarding the inflammatory process close to the nerve roots or in the pain pathways.

\section{Conclusions}

Chronic lumbar radicular pain may be associated with a high level of the pro-inflammatory TNF- $\alpha$ in serum after disc herniation, and of anti-inflammatory cytokine IL-4 in patients with lumbar radiculopathy, which possibly indicate a favorable outcome. This is the first demonstration of a positive correlation between pain and pro-inflammatory cytokines, and negative correlation between pain and anti-inflammatory cytokines.

\section{Conflict of Interest}

No potential conflict of interest relevant to this article was reported.

\section{Acknowledgments}

The work was supported by the National Natural Science Foundation of China (81171173). The authors gratefully acknowledge the support of Shu Yang, who assisted with participant recruitment, and Bao-Yi Zhou, who assisted with data collection and with ELISA assays.

\section{References}

1. Ropper AH, Zafonte RD. Sciatica. N Engl J Med 2015;372:1240-8.

2. Dagenais S, Caro J, Haldeman S. A systematic review of low back pain cost of illness studies in the United States and internationally. Spine J 2008;8:8-20.

3. Fleury G, Nissen MJ, Genevay S. Conservative treatments for lumbar radicular pain. Curr Pain Headache Rep 2014;18:452.

4. de Souza Grava AL, Ferrari LF, Defino HL. Cytokine inhibition and time-related influence of inflammatory stimuli on the hyperalgesia induced by the nucleus pulposus. Eur Spine J 2012;21:537-45.

5. Brisby H, Olmarker K, Larsson K, Nutu M, Rydevik B. Proinflammatory cytokines in cerebrospinal fluid and serum in patients with disc herniation and sciatica. Eur Spine J 2002;11:62-6.

6. Shamji MF, Setton LA, Jarvis W, et al. Proinflammatory cytokine expression profile in degenerated and herniated human intervertebral disc tissues. Arthritis Rheum 2010;62:1974-82.

7. Uceyler N, Riediger N, Kafke W, Sommer C. Differential gene expression of cytokines and neurotrophic factors in nerve and skin of patients with peripheral neuropathies. J Neurol 2015;262:203-12.

8. Zelenka M, Schafers M, Sommer C. Intraneural injection of interleukin-1beta and tumor necrosis factor-alpha into rat sciatic nerve at physiological doses induces signs of neuropathic pain. Pain 2005;116:25763.

9. Wagner R, Janjigian M, Myers RR. Anti-inflammatory interleukin-10 therapy in CCI neuropathy decreases thermal hyperalgesia, macrophage recruitment, and endoneurial TNF-alpha expression. Pain 1998;74:35-42.

10. Zanella JM, Burright EN, Hildebrand K, et al. Effect of etanercept, a tumor necrosis factor-alpha inhibitor, on neuropathic pain in the rat chronic constriction 
injury model. Spine (Phila Pa 1976) 2008;33:227-34.

11. Ohtori S, Miyagi M, Eguchi Y, et al. Epidural administration of spinal nerves with the tumor necrosis factor-alpha inhibitor, etanercept, compared with dexamethasone for treatment of sciatica in patients with lumbar spinal stenosis: a prospective randomized study. Spine (Phila Pa 1976) 2012;37:439-44.

12. Di Martino A, Merlini L, Faldini C. Autoimmunity in intervertebral disc herniation: from bench to bedside. Expert Opin Ther Targets 2013;17:1461-70.

13. McCoy MK, Tansey MG. TNF signaling inhibition in the CNS: implications for normal brain function and neurodegenerative disease. J Neuroinflammation 2008;5:45.

14. Andrade P, Hoogland G, Del Rosario JS, Steinbusch HW, Visser-Vandewalle V, Daemen MA. Tumor necrosis factor- $\alpha$ inhibitors alleviation of experimentally induced neuropathic pain is associated with modulation of TNF receptor expression. J Neurosci Res 2014;92:1490-8.

15. Uceyler N, Rogausch JP, Toyka KV, Sommer C. Differential expression of cytokines in painful and painless neuropathies. Neurology 2007;69:42-9.

16. Pedersen LM, Schistad E, Jacobsen LM, Roe C, Gjer- stad J. Serum levels of the pro-inflammatory interleukins 6 (IL-6) and -8 (IL-8) in patients with lumbar radicular pain due to disc herniation: a 12-month prospective study. Brain Behav Immun 2015;46:1326.

17. Kraus J, Borner C, Giannini E, et al. Regulation of mu-opioid receptor gene transcription by interleukin-4 and influence of an allelic variation within a STAT6 transcription factor binding site. J Biol Chem 2001;276:43901-8.

18. Opal SM, DePalo VA. Anti-inflammatory cytokines. Chest 2000;117:1162-72.

19. Sekiguchi M, Sekiguchi Y, Konno S, Kobayashi H, Homma Y, Kikuchi S. Comparison of neuropathic pain and neuronal apoptosis following nerve root or spinal nerve compression. Eur Spine J 2009;18:197885.

20. Czeschik JC, Hagenacker T, Schafers M, Busselberg D. TNF-alpha differentially modulates ion channels of nociceptive neurons. Neurosci Lett 2008;434:293-8.

21. Wang H, Schiltenwolf M, Buchner M. The role of TNF-alpha in patients with chronic low back pain-a prospective comparative longitudinal study. Clin J Pain 2008;24:273-8. 